\title{
Resistivity and dissipation in pulsar magnetospheres
}

\author{
Jason Li ${ }^{1}$, Anatoly Spitkovsky ${ }^{1}$, and Alexander Tchekhovskoy ${ }^{2}$ \\ ${ }^{1}$ Department of Astrophysical Sciences, Princeton University, \\ Peyton Hall, Princeton, NJ 08544, USA \\ email: jgli@astro.princeton.edu \\ ${ }^{2}$ Princeton Center for Theoretical Science, Princeton University, \\ Jadwin Hall, Princeton, NJ 08544, USA
}

\begin{abstract}
Current models of pulsar magnetospheres typically assume either a complete absence of plasma or abundant ideal plasma filling the magnetosphere in order to compute the field structure. The latter condition is thought to be closer to reality, but we know of a number of pulsars in which the ideal conditions break down, resulting in dissipation and high-energy emission. In this work we formulate a resistive force-free scheme that allows us to consider the effects of resistive plasma and accelerating fields on the magnetospheric structure. We run numerical simulations to construct a family of resistive solutions that smoothly bridges the gap between the vacuum and the force-free magnetosphere solutions. We further provide a selfconsistent model for the spin-down of intermittent pulsars, pulsars which appear to transition between radio-loud and radio-quiet states with different spin-down rates. Finally, we present models for high-energy emission from reconnecting current sheets in Gamma-ray pulsars.
\end{abstract}

Keywords. pulsars: general, gamma rays: theory, MHD, acceleration of particles

\section{Introduction}

Until very recently, quantitative solutions of the global pulsar magnetospheric structure existed only for the vacuum limit (Deutsch 1955) and for the limit of abundant plasma in force-free electrodynamics (see e.g., Contopoulos et al. 1999; Spitkovsky 2006). The real pulsar magnetosphere is likely operating somewhere in between these limits, with various accelerating gaps, regions of pair production, and strong current sheets likely causing local violations of the ideal MHD constraint, $\mathbf{E} \cdot \mathbf{B}=0$. Knowing the structure of the magnetosphere, including such non-ideal effects, would be very useful for calculating the properties of pulsar emission. Indeed, currently the ideal force-free models that include the back-reaction of plasma currents on the field structure lack any accelerating fields by construction, and thus cannot be used to directly predict the spectra of gamma-ray radiation observed by the Fermi GST. Recently, we have developed a resistive force-free method that allows for variations in plasma supply and accelerating electric fields ( $\mathrm{Li}$ et al. 2012a; see also Kalapotharakos et al. 2012). We specify an Ohm's Law $\mathbf{j}^{\prime}=\sigma \mathbf{E}^{\prime}$ in the $\mathbf{E} \times \mathbf{B}$ drift frame of the massless plasma, representing a current sourced by relative counterstreaming of opposite charges along the magnetic field in this minimal velocity bulk fluid frame. This Ohm's Law provides a closure for Maxwell's Equations and allows us to construct a continuum of solutions transitioning between the vacuum and ideal force-free solutions as the conductivity parameter $\sigma$ is varied between 0 and $\infty$. The freedom in choosing the conductivity of the plasma further allows us to construct quantitative models for the spin-down of intermittent pulsars and explore the high-energy emission from $\gamma$-ray pulsars. 


\section{Intermittent Pulsars}

A number of recently discovered "intermittent" pulsars switch between two distinct states: an "on", radio-loud state, and an "off", radio-quiet state. Spin-down rates in the two states differ by a large factor, 1.5 - 2.5 (Kramer et al. 2006; Lyne 2009; Camilo et al. 2012; see also Lyne et al. (2010) for possibly related mode changing in radio pulse profiles). This is not easily understood in the context of current models since the forcefree to vacuum spin-down ratio is always greater than 3. In Li et al. (2012b) we model the "on" state as a nearly ideal force-free magnetosphere with abundant magnetospheric plasma supply able to generate coherent radio emission. The lack of radio emission in the "off" state is associated with plasma supply disruption that results in lower plasma density on the open field lines. We model the "off" state using nearly vacuum conditions on the open field lines and nearly ideal force-free conditions on the closed field lines, where plasma can remain trapped even in the absence of pair production. Figure 1 illustrates magnetic field lines in the $\mu-\boldsymbol{\Omega}$ plane for these two states, both with $60^{\circ}$ inclination angle. Color is representative of the out-of-plane magnetic field. The left panel, the "on" state, is a resistive solution taken at high conductivity, and is essentially force-free-like but with a spatially resolved current sheet. The right panel shows the "off" state, with high conductivity in the closed zone interior to the red contour, and low conductivity on open field lines exterior to the red contour.
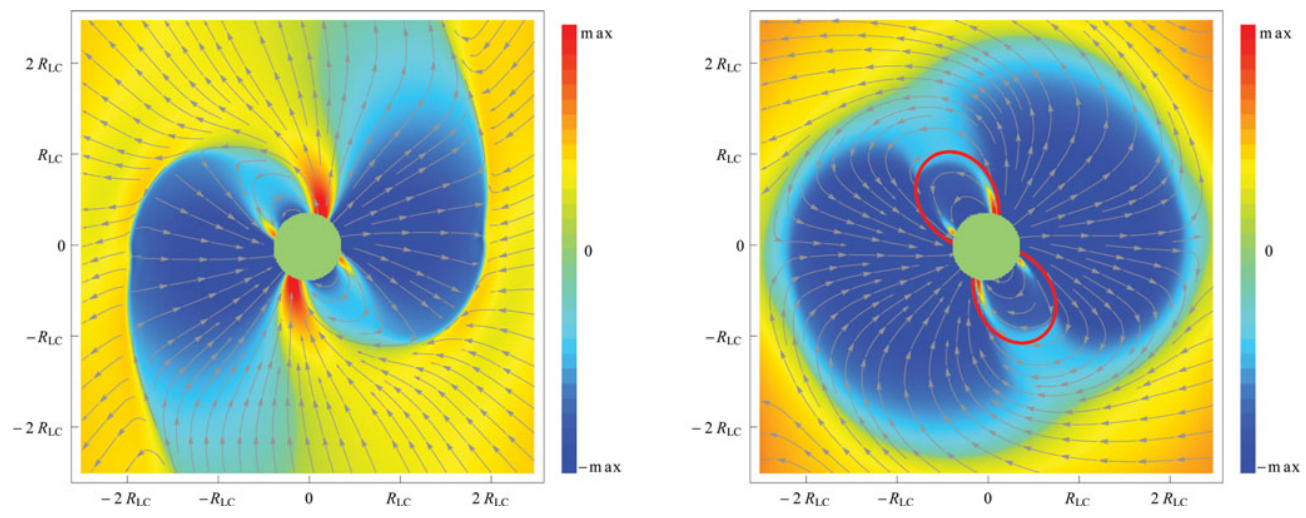

Figure 1. Magnetic fields in $\mu-\boldsymbol{\Omega}$ plane. Color is out-of-plane field. Left: Intermittent pulsar "on" state, highly conducting everywhere. Right: Intermittent pulsar "off" state, resistive on open field lines, highly conducting in closed zone.

In the "off" state, current from toroidal advection of charged plasma in the closed zone leads to greater magnetic flux passing through the light cylinder and a larger fraction of open field lines than in the vacuum solution. Since open field lines carry Poynting flux, the "off" state spin-down is larger than the vacuum spin-down value. It turns out that the "off" state spin-down is a factor of $\sim 2$ higher than vacuum values for all inclination angles, and we naturally obtain a range of spin-down ratios between the "on" and "off" states, $\sim 1.2-2.9$, which corresponds to a likely range of pulsar inclination angles, $30-90^{\circ}$ (Li et al. 2012b).

\section{Gamma-ray pulsars}

Our resistive force-free method can spatially resolve the accelerating electric fields in the pulsar current sheet, offering the exciting prospect of explaining high-energy emission from the current sheet (e.g., Lyubarskii 1996). There is an ambiguity in determining 
particle velocities parallel to the magnetic field, however, as in all force-free methods, and we still have some freedom to choose particle velocities, especially in the current sheet. The characteristic double peaked $\gamma$-ray light curves suggest that particle motions in the sheet are nearly radially outwards, but possibly have a small toroidal component, representing angular momentum lost by the pulsar. At present it is not completely clear at what radial distance from the pulsar the $\gamma$-rays come from. We discuss two models for the $\gamma$-ray emission: near the light cylinder $R_{\mathrm{LC}}=c / \Omega$, and in the wind zone beyond a few $R_{\mathrm{LC}}$.

The primary argument in favor of $\gamma$-ray emission coming from near $R_{\mathrm{LC}}$ is that the available magnetic energy density decreases with radius, $u_{B}=B^{2} / 8 \pi \propto 1 / R^{2}$. The observed interpulse bridge emission also arises quite naturally from particles flying along open magnetic field lines near the current layer, interior to $R_{\mathrm{LC}}$, as shown explicitly by Bai \& Spitkovsky (2010). Emission from near the current sheet or the current sheet itself, beamed roughly in the radial direction, can give rise to a strong caustic in the skymap and double peaked light curves (Bai \& Spitkovsky 2010; Arka \& Dubus 2012). We have explored a number of possible methods for producing this necessary beaming by studying microphysical processes operating in the current sheet ( $\mathrm{Li}$ et al. 2012c, in prep). One possibility is that particles fly outwards along all open field lines and nearly radially outwards in the laboratory frame. Particles only emit near the current sheet or layer, however, where they become heated and can gyrate around magnetic field lines, radiating synchrotron photons radially outwards. Another possibility is that particles are ejected relativistically in plasmoids from local $\mathrm{x}$ points in the current sheet. Plasmoids will approximately follow the magnetic field direction on either side of the sheet, so they travel radially outwards away from the Y-surface in the laboratory frame. The accelerating electric field in the current sheet can also play an important dynamical role. The electromagnetic fields carry angular momentum, as the pulsar is spinning down, and the accelerating fields can drive particles in the current sheet in the toroidal direction. The strong $\gamma$-ray caustic will then be shifted to earlier phases closer to the radio emission, depending on the strength of the accelerating electric fields, in accordance with the observed light curves.

The wind zone beyond a few light cylinder radii offers an alternative solution (see e.g., Kirk et al. 2002; Petri \& Kirk 2005; Petri 2011; Petri 2012; Li et al. 2012c, in prep), whose biggest advantage is that radial beaming of emission arises quite naturally. Beyond a few $R_{\mathrm{LC}}$, the force-free drift velocity (Bogovalov 1999) satisfies $v_{\phi} / v_{r}=R_{\mathrm{LC}} / R$, i.e., the velocity is directed radially outward, with wind $\Gamma=\sqrt{1+\left(R / R_{\mathrm{LC}}\right)^{2}}$. Any microphysical process driving particles to high transverse velocities along $\vec{E}$ or $\vec{B}$ in the wind frame will have transverse velocities suppressed by $\Gamma$ in the observer frame, with maximum magnitude $c / \Gamma$. Several authors have suggested that sufficiently high lorentz factors to generate $\mathrm{GeV}$ emission can be reached in the synchrotron radiation reaction limit, where reconnection heating balances synchrotron cooling losses (e.g., Uzdensky \& Spitkovsky 2012).

Figure 2 gives the skymap and light curves when radiation is beamed in the radial direction from beyond $2 R_{\mathrm{LC}}$, for $60^{\circ}$ rotators and using the field solutions from our highly conducting force-free-like model. Radio emission occurs near $\xi_{\text {obs }}=60$ and $\phi=0$. The strong caustic essentially traces the shape of the current sheet on the skymap, accounting for light travel time delay effects. Strongly doubled peaked light curves will be seen for a range of observer angles, but two immediate questions stand out. First, there is no bridge emission, and it is unclear how to produce it in the wind zone, where all the plasma is flying radially outwards at relativistic speeds. It may be necessary to 


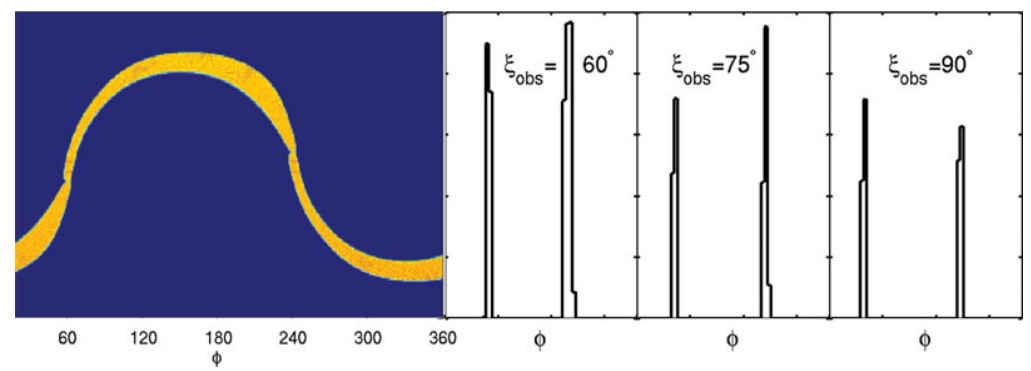

Figure 2. Skymap for wind zone model with emission from the current sheet beyond $2 R_{\mathrm{LC}}$. Pulsar inclination angle is 60 degrees.

produce this emission closer in to the light cylinder, where $\Gamma$ is smaller and beaming is less strong. A second issue is that in the wind zone it is more difficult to shift the caustic to earlier phases than closer in to the light cylinder, because toroidal particle velocities fall as $v_{\phi} \propto 1 / R$ for constant angular momentum. A better understanding of microphysical reconnection processes in the wind frame may help to address both of these issues.

To summarize, we believe we have a good first order picture of the motions of $\gamma$ ray emitting particles in the context of reconnection models of high-energy emission. The observed light curves suggest that particles travel radially outward in the current sheets, with second order corrections to the velocities in the toroidal direction. Recently developed resistive methods give some hope for incorporating these corrections into microphysical reconnection models, and determining the radii at which emission occurs. Ultimately, particle-in-cell or magnetohydrodynamical simulations of the global pulsar structure, or the local current sheet physics, could provide additional information about particle acceleration and high-energy radiation.

\section{References}

Arka, I. \& Dubus, G. 2012, arXiv:1208.2819

Bai, X. \& Spitkovsky, A. 2010, ApJ, 715, 1282

Bogovalov, S. V. 1999, Astronomy \& Astrophysics, 349, 1017

Camilo, F., Ransom, S. M., Chatterjee, S., Johnston, S., \& Demorest, P. 2012, ApJ, 746, 63

Contopoulos, I., Kazanas, D., \& Fendt, C. 1999, ApJ, 511, 351

Deutsch, A. J. 1955, Annales d'Astrophysique, 18, 1

Kalapotharakos, C., Kazanas, D., Harding, A., \& Contopoulos, I. 2012, ApJ, 749, 2

Kirk, J. G., Skjæraasen, O., \& Gallant, Y. A. 2002, A\&3A, 388, L29

Kramer, M., Lyne, A. G., O’Brien, J. T., Jordan, C. A., \& Lorimer, D. R. 2006, Science, 312, 549

Li, J., Spitkovsky, A., \& Tchekhovskoy, A. 2012a, ApJ, 746, 60

Li, J., Spitkovsky, A., \& Tchekhovskoy, A. 2012b, ApJL, 746, L24

Li, J., Spitkovsky, A., \& Tchekhovskoy, A. 2012c, in prep

Lyne, A. G. 2009, ASSL, 357, 67

Lyne, A., Hobbs, G., Kramer, M., Stairs, I., \& Stappers, B. 2010, Science, 329, 408

Lyubarskii, Y. E. 1996, $A \mathscr{G} A, 311,172$

Pétri, J. 2011, MNRAS, 412, 1870

Pétri, J. 2012, MNRAS, 424, 2023

Pétri, J. \& Kirk, J. G. 2005, ApJL, 627, L37

Spitkovsky, A. 2006, ApJL, 648, L51

Uzdensky, D. A. \& Spitkovsky, A. 2012, arXiv:1210.3346 